\title{
Erratum: Precision Mass Measurements on Neutron-Rich Rare-Earth Isotopes at JYFLTRAP: Reduced Neutron Pairing and Implications for $r$-Process Calculations [Phys. Rev. Lett. 120, 262701 (2018)]
}

M. Vilen๑, J. M. Kelly, A. Kankainen, M. Brodeur, A. Aprahamian, L. Canete, T. Eronen, A. Jokinen, T. Kuta, I. D. Moore, M. R. Mumpower, D. A. Nesterenko, H. Penttilä, I. Pohjalainen, W. S. Porter, S. Rinta-Antila, R. Surman,

A. Voss, and J. Äystö

(Received 15 August 2019; published 24 March 2020)

DOI: 10.1103/PhysRevLett.124.129901

A recent remeasurement of the ${ }^{163} \mathrm{Eu}$ and ${ }^{163} \mathrm{Gd}$ mass excesses [1] by our group, using ${ }^{133} \mathrm{Cs}^{+}$and ${ }^{136} \mathrm{Xe}^{+}$as calibrants, respectively, revealed that the ${ }^{163} \mathrm{Dy}^{+}$calibrant used in the measurement reported in [2] was incorrectly assigned. Following a detailed analysis presented in [1], it was found that the calibrant was most likely ${ }^{146} \mathrm{La}^{16} \mathrm{O}^{1} \mathrm{H}^{+}$, or its mixture with ${ }^{163} \mathrm{Dy}^{+}$.

During the same campaign [1] the mass excess of ${ }^{162} \mathrm{Eu}$ was also remeasured since the type and duration of the excitation for the measurement reported in [2] did not allow to resolve a low-lying isomeric state reported in [3]. The new JYFLTRAP measurements [1] were done both with the phase-imaging ion cyclotron resonance technique and also using a longer excitation time $(1600 \mathrm{~ms})$ in the time-of-flight ion cyclotron resonance measurements. The new ground and isomeric state mass-excess values of ${ }^{162} \mathrm{Eu}$ and ${ }^{162} \mathrm{Eu}^{m}$ reported in [1] agree with [3].

In conclusion, the mass-excess values of ${ }^{162,163} \mathrm{Eu}$, and ${ }^{163} \mathrm{Gd}$ from [1] should be considered as superseding the ones from [2]. In [2], ${ }^{163} \mathrm{Eu}$ and ${ }^{163} \mathrm{Gd}$ made use of an incorrectly assigned calibrant, and the ${ }^{162} \mathrm{Eu}$ mass excess was a mixture of both ground state and isomeric state due to the type and length of the excitation used. We note that the changes in the mass-excess values of ${ }^{162,163} \mathrm{Eu}$, and ${ }^{163} \mathrm{Gd}$ do not change the conclusions presented in [2]. The $S_{2 n}$ values do not support a subshell closure at $N=100$ (see Fig. 1) and neutron pairing has been found to be weaker than predicted by the theoretical models (see Fig. 2). The $S_{n}$ values change only a little with the revised ${ }^{162,163} \mathrm{Eu}$ and ${ }^{163} \mathrm{Gd}$ mass values, and hence the impact on the $r$ process abundances is small. For details, see [1].

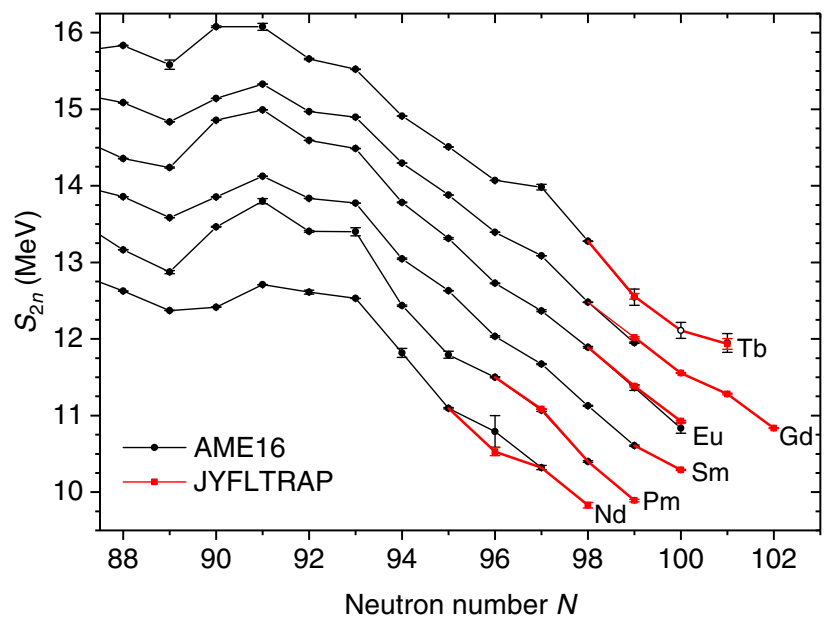

FIG. 1. Figure 2 of [2] with the revised mass-excess values of ${ }^{162,163} \mathrm{Eu}$, and ${ }^{163} \mathrm{Gd}$. Two-neutron separation energies $S_{2 n}$ from this work (red) together with the experimental (solid black circles) values and an extrapolated value for ${ }^{165} \mathrm{~Tb}$ (open black circle) from AME16 [4]. 


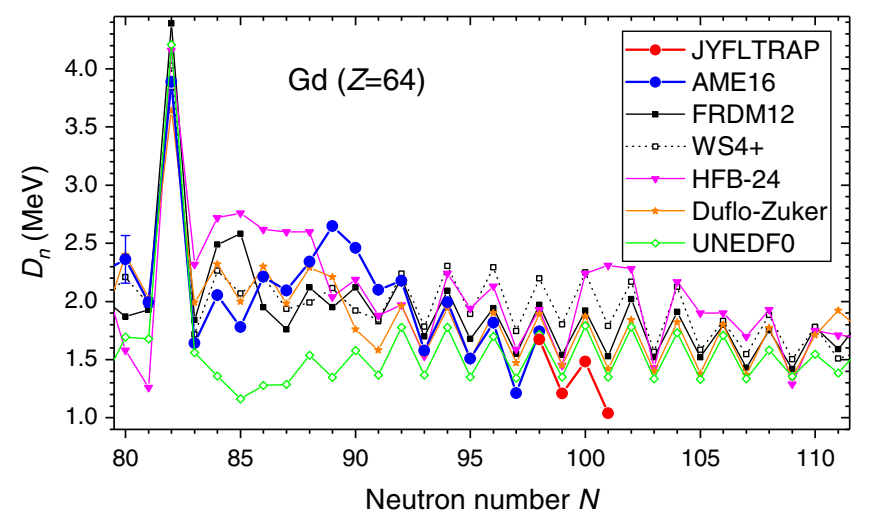

FIG. 2. Figure 3 of [2] with the revised mass-excess value of ${ }^{163} \mathrm{Gd}$. Neutron pairing energies from this work (red circles) and AME16 (blue) in comparison with various theoretical predictions for the $\mathrm{Gd}$ isotopes.

[1] M. Vilen, J. Kelly, A. Kankainen, M. Brodeur, A. Aprahamian, L. Canete, R. de Groote, A. de Roubin, T. Eronen, A. Jokinen, I. Moore, M. Mumpower, D. Nesterenko, J. O'Brien, A. P. Perdomo, H. Penttilä, M. Reponen, S. Rinta-Antila, and R. Surman, related paper, Phys. Rev. C 101034312 (2020).

[2] M. Vilen, J. M. Kelly, A. Kankainen, M. Brodeur, A. Aprahamian, L. Canete, T. Eronen, A. Jokinen, T. Kuta, I. D. Moore, M. R. Mumpower, D. A. Nesterenko, H. Penttilä, I. Pohjalainen, W. S. Porter, S. Rinta-Antila, R. Surman, A. Voss, and J. Äystö, Phys. Rev. Lett. 120, 262701 (2018).

[3] D. J. Hartley et al., Phys. Rev. Lett. 120, 182502 (2018).

[4] M. Wang, G. Audi, F. Kondev, W. Huang, S. Naimi, and X. Xu, Chin. Phys. C 41, 030003 (2017). 